EVS27

Barcelona, Spain, November 17-20, 2013

\title{
Configuration Analysis of Plug-in Hybrid Systems using Global Optimization
}

\author{
Insup Kim ${ }^{1}$, Hyunsup Kim \\ ${ }^{1}$ Hyundai-Kia Motors, 150 HyundaiYeonguso-ro Hwaseong-si Gyeonggi-do Republic of Korea, monjey@hyundai.com
}

\begin{abstract}
The purpose of the study is to analyze the configurations of Plug-in Hybrid Electric Vehicles (PHEV) with respect to fuel economy. Existing studies mostly focus on hybrid systems or few PHEV systems by only considering power split ratio and component efficiency. This paper adds original contribution to these literatures. First of all, this study compares and analyzes "series $+\boldsymbol{\alpha}$ ” PHEV - Input split, Series-output split and Series-parallel, which is consisted of a single Planetary gear or spur gear and clutches. Those are currently applied to mass-production vehicles such as Toyota Prius PHEV, Chevrolet Volt and Honda Accord PHEV. On top of that, it examines the impact of the transmission mechanical losses on Dynamic programming (DP) results and especially the planetary gear loss is modelled using power split ratio analysis. Lastly, the effect of Series mode for each PHEV system is examined by analysis of the theoretical system efficiency and DP in a certain driving profile. From this study the strength and weakness of PHEV systems are revealed depending on a driving condition and battery status, e.g. charging depleting (CD) or charging sustaining (CS). The PHEV system analysis in this study can help select proper system for a certain purpose.
\end{abstract}

Keywords: modelling, optimization, PHEV (Plug in hybrid electric vehicle), series HEV, simulation

\section{Introduction}

In the United States, President Obama has called for manufacturers of cars and trucks to double their fuel economy by 2025 , to a lofty 54.5 miles per gallon $(4.3 \mathrm{~L} / 100 \mathrm{~km}$, or $23.2 \mathrm{~km} / \mathrm{l})$. The European Union targets a 40 percent reduction in automotive $\mathrm{CO}_{2}$ emission by 2018: the target of 130 grams $\mathrm{CO}_{2}$ of per kilometre driven equates to $5.6 \mathrm{~L} / 100 \mathrm{~km}$ (42 mpg or $17.9 \mathrm{~km} / \mathrm{l}$ ) [1]. In order to meet environmental targets for fuel economy and/or $\mathrm{CO}_{2}$ emissions, the automakers have no choice but to develop Plug-in Hybrid Vehicle (PHEV) or Electric Vehicle (EV). Because of range limitation of EV, PHEV has been prevalent while still allowing the usual freedom to travel.

Automakers have been focusing on developing a competitive PHEV by introducing a variety of system architectures. Chinese automaker BYD Auto released the F3DM, which had Seriesparallel system, in the Chinese market in 2008. In 2011, Fisker Automotive introduced a Series PHEV, Fisker Karma and in the same year General Motors (GM) introduced Volt, which had Seriesoutput Power split system (S/O) with one planetary gear, three clutches. And in 2012 Toyota Motor launched Prius PHEV, which had Input split (I/S) system that was similar to a previous hybrid system and had a bigger battery. In 2013, Honda 
Accord PHEV with Series-Parallel system (S/P) was released.

Table1: Models of PHEVs

\begin{tabular}{|c|c|c|c|}
\hline Manufacturer & Model & System & $\begin{array}{c}\text { CD FE } \\
\text { (MPGe) }\end{array}$ \\
\hline Fisker & Fisker Karma & Series & 52 \\
\hline BYD & F3DM & Series-Parallel & 88 \\
\hline GM & Volt & $\begin{array}{c}\text { Series- } \\
\text { Output Split }\end{array}$ & 98 \\
\hline Toyota & Prius PHEV & Input Split & 95 \\
\hline Honda & Accord PHEV & Series-Parallel & 115 \\
\hline
\end{tabular}

While it has high system efficiency in electric driving mode with low mechanical drag, Series system has low efficiency in hybrid driving mode because of electric machines loss. Consequently, in order to overcome low system efficiency in hybrid driving mode, series system needs to have additional transmission mode - Power Split or Parallel - by adding clutch or gearset. Additional transmission mode provides higher systems efficiency since part of engine directly connects to output shaft, which has no transfer loss. For adding Power Split or Parallel mode, however, mechanical elements - clutch or gear set - should be added and this leads to increase mechanical losses. Consequently electric transfer loss as well as mechanical element loss should be considered to figure out which Plug-in system has better system efficiency.

The control technology and system efficiency of hybrid systems are analysed and compared by using system analysis and forward-looking simulation [2]-[4]. And the most common hybrid powertrain structures - Parallel and Power split were compared taking in account fuel and electrical consumption by using dynamic programming and rule-based control method [5][7]. In line with 2-mode hybrid system introduction, input, output and compound split schemes are assessed in terms of fuel economy and vehicle performance analytically [8]. These literatures provides the control policy and system analysis only for HEV by using DP and forward simulation. As PHEV is becoming prevalent, the literature about PHEV is increasing as well. A. Da Costa investigate the impact of driving conditions and standard test procedure on the true benefits of PHEVs - Parallel, Input split, Output split and Series - for Europe and the US market [9]. Input split and Series-output split system are compared by using dynamic model and control algorithm for each PHEV [10]. These literatures, however, study a limited number of
PHEV, do not include new plug-in system, Seriesparallel and only consider system characteristic and motor/generator efficiency. In this study the most common plug-in hybrid systems - Input split, Series-output split and Series-parallel - are modelled and analysed, and in particular transmission mechanical loss are also modelled and applied to DP.

First of all, in the steady state condition (constant speed), the system efficiency - Series-path efficiency and transmission mechanical efficiency - is compared by the theoretical analysis. On top of that in standard driving cycles - UDDS, HWFET and US06 - the system efficiency of each system is compared by using dynamic programming. In conclusion the system efficiency characteristic of PHEV system can be found under a certain driving condition.

\section{PHEV powertrain topologies}

Two types of HEV and PHEV topology that can be found are parallel ones and series ones. The energy-flow diagram of a general HEV/PHEV powertrain is shown in Figure 2. A parallel PHEV structure is obtained by deleting path $\mathrm{P}_{\mathrm{N}}$ and $\mathrm{P}_{\mathrm{G}}$. $\mathrm{A}$ series HEV structure does not contain the path $\mathrm{P}_{\mathbf{C}}$ or the gear and clutch block. Combined PHEV may require that some nodes or paths be physically linked. For example, Series-parallel system and Input split system has a series path (path $\mathrm{P}_{\mathbf{N}}$ and $\mathrm{P}_{\mathbf{G}}$ ) and a parallel path (path $\mathrm{P}_{\mathbf{C}}$ ) by adding a clutch and planetary gear set respectively.

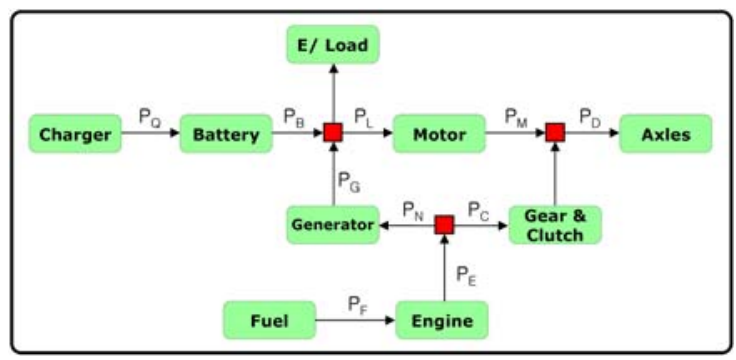

Figure2: Energy flow of a general HEV/ PHEV [11]

Series structures are advantageous with respect to pollutant emissions and mechanical drag, but have serious disadvantages with respect to fuel economy. Parallel and combined HEV can achieve both excellent fuel economy and low pollutant emissions. Therefore parallel and combined structures are prevalent for HEV [11].

When it comes to PHEV, however, Series structures are advantageous with respect to high efficiency in EV due to simpler transmitting path. 
Therefore on Charging depleting (CD) cycle of PHEV, Series can be better than Parallel. For this reason, PHEV puts more weigh on Series and combine parallel or power split modes by adding clutch or gear for Charging sustaining (CS) cycle. In this paper any Plug-in hybrid systems with series and parallel or power-split, are included in a combined topologies [12].

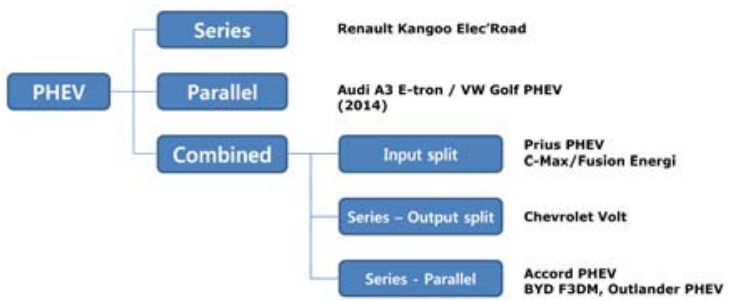

Figure3: PHEV powertrain topologies

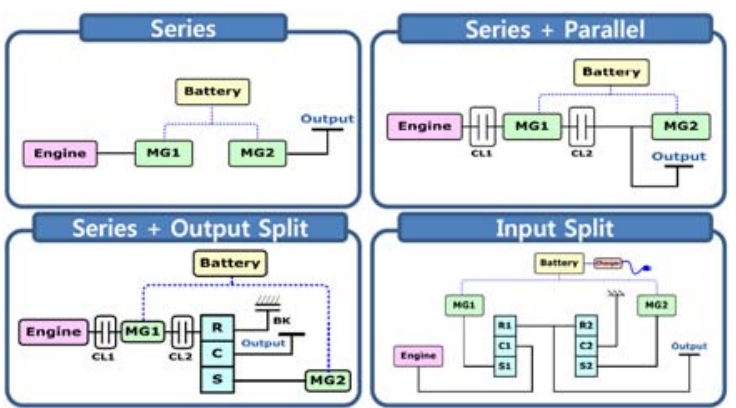

Figure1: Configurations of (Series $+\boldsymbol{\alpha})$ PHEVs

While it has high system efficiency in electric driving mode with low mechanical drag, Series system has low efficiency in hybrid driving mode because of electric machines loss. Consequently in order to overcome low system efficiency in hybrid driving mode, the system need to have additional transmission mode - Power Split or Parallel - by adding clutch or gear set. Additional transmission mode provides higher systems efficiency since part of engine directly connects to output shaft, which has no transfer loss. For adding Power Split or Parallel mode, however, mechanical elements - clutch or gear set - should be added and this leads to increase mechanical losses. Consequently electric transfer loss as well as mechanical element loss should be considered to figure out which Plug-in system has better system efficiency. For a hybrid vehicle application, parallel and Input split topology are most prevalent, because in the case of parallel ones it is easy to modify from conventional vehicle to hybrid one, and input split has a higher system efficiency especially in electric driving condition plus can have the small size of the components compared to the series topology.
For a plug-in hybrid, on the other hand, electric driving range by one charge is so critical that Series based topologies, which have better transmission efficiency in EV owing to short mechanical path from motor to wheel, have an advantage.

\subsection{Input split system}

Input split is often described as part series and part parallel. It is consisted of two planetary gears power split gear and motor reduction gear - and two motors. The ratio of series to parallel determined by a planetary gear ratio.



Figure4: Configuration of Input split system

Since the engine is not directly connected to the wheels the engine speed can operate independently of vehicle speed and torque. The speed of generator is determined by vehicle speed and engine speed, and the torque of that is determined by engine torque. Speed and torque equations of Input split are below:

$$
\begin{aligned}
& T_{M G 1}=-\frac{1}{A+1} T_{\text {Eng }} \\
& \boldsymbol{T}_{\text {Out }=}=\frac{\boldsymbol{A}}{\boldsymbol{A}+1} \boldsymbol{T}_{\text {Eng }}+\boldsymbol{B} \times \boldsymbol{T}_{\boldsymbol{M G} 2} \\
& W_{\text {Eng }}=\frac{1}{A+1} W_{M G 1}+\frac{A}{A+1} W_{\text {Out }}
\end{aligned}
$$

Where $A$ is the ratio of gear teeth between the sun and the ring of a power split planetary gear and $\mathrm{B}$ is the ratio of gear teeth between the sun and the ring of a motor planetary gear. The examples of Input split PHEVs are Toyota Prius PHEV and Ford Fusion Energi.

\subsection{Series - Output split system}

Series-output split can also be classified as an EREV that is a vehicle that functions as a fullperformance battery electric vehicle when energy is available from an onboard RESS (Rechargeable Energy Storage System) and having an auxiliary energy supply that is only engaged when the RESS energy is not available [13]. Series-output provides two EV operations (One-Motor EV, Two-Motor 
EV) and two extended-range operations (Series and Output spit). It operates with Series at low driving speeds and with Output split at high speeds and lighter loads [14]. Adding Output split provides a better system efficiency over Series, however it requires an additional mechanical components, two clutches and one brake, which add mechanical loss as well.

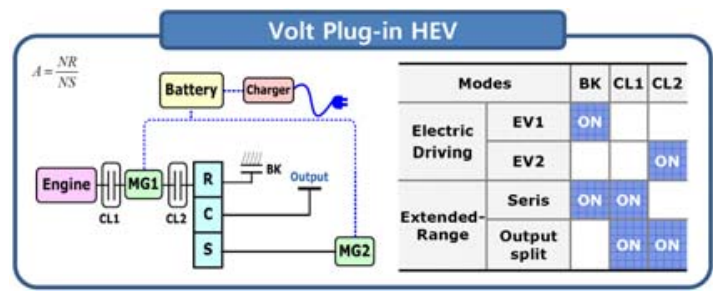

Figure5: Configuration of Series-Output split system

Speed and torque equations of Series-output split are below:

$$
\begin{aligned}
\text { EV / Series }: T_{M G 2}= & \frac{A}{A+1} T_{\text {Out }}, w_{M G 2}=(A+1) \times w_{\text {Out }} \\
\text { Output }- \text { split }: T_{\text {Out }}= & \frac{A+1}{A}\left(T_{\text {Eng }}+T_{M G 1}\right), T_{M G 2}=\frac{1}{A+1} T_{\text {Out }} \\
& w_{M G 2}+A \times w_{\text {Eng }}=(A+1) \times w_{\text {Out }}
\end{aligned}
$$

Where A is the ratio of gear teeth between the sun and the ring of a planetary gear. The examples of Series-Output split PHEV is GM Chevrolet Volt.

\subsection{Series - Parallel system}

Series-parallel overcomes a disadvantage of series by providing parallel mode, which the engine is directly connected to the wheels, at a high speeds and lighter loads. During low-load condition such as launching or city driving, EV is mainly selected and the driving mode is switched to Series for the acceleration during normal-load or heavy-load condition. In order to provide parallel mode, one clutch is needed and this add mechanical loss compared to a series-only system.

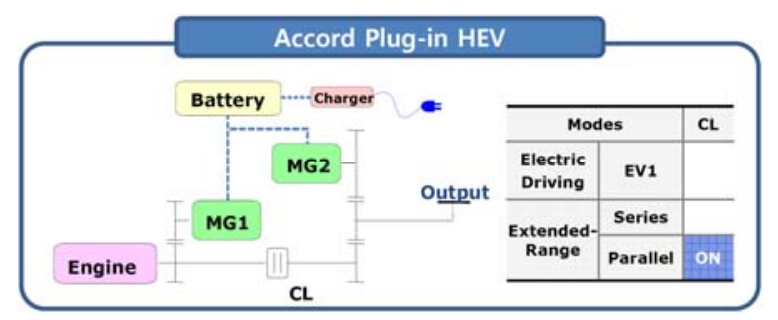

Figure6: Configuration of Series-Parallel system

$$
\begin{gathered}
E V / \text { Series : } T_{M G 2}=\frac{1}{R 3} T_{\text {Out }}, T_{M G 1}=-R 1 \times T_{E n g}, \\
w_{M G 2}=R 3 \times w_{\text {Out }} \\
\text { Parallel : } T_{\text {Out }}=\left(T_{E n g}+T_{M G 1} / R 1\right) \times R 2+T_{M G 2} \times R 3 \\
w_{E n g}=R 2 \times w_{\text {Out }}
\end{gathered}
$$

Where R1 is the ratio of gear teeth between the engine and the generator, $\mathrm{R} 2$ is the ratio of gear teeth between the engine and the output, and R3 is the ratio of gear teeth between the motor and the output. The example of Series-parallel PHEV is Honda Accord PHEV.

\section{Vehicle Modelling and Dynamic Programming}

In order to compare fuel economy of PHEVs systems, same vehicle specification, final gear and motor/ generator efficiency are assumed and analytical method and dynamic programming algorithm are used. First of all, the components of vehicle - engine, two electric machines and high voltage battery - are modelled. As well as the transmission mechanical losses - gear loss, clutch loss and oil pump drag - are considered.

\subsection{Vehicle Modelling}

It is assumed that each PHEV system has a same vehicle specification - road load coefficients, tyre radius and weight, engine, battery and motor except transmission. Transmission gear ratio are used as same as a mass-production PHEVs - Prius PHEV, Volt and Accord PHEV.

Table2: PHEVs specifications

\begin{tabular}{|c|c|}
\hline $\begin{array}{c}\text { Road Load } \\
\text { (f0 / f1 / f2) }\end{array}$ & $125.44 \mathrm{~N} / 0 / 0.4 \mathrm{~N} /(\mathrm{m} / \mathrm{s})^{2}$ \\
\hline Weight & $1588 \mathrm{~kg}$ \\
\hline Tyre radius & $0.305 \mathrm{~m}$ \\
\hline Engine & GSL 1.6L, 82kW \\
\hline Battery & LiPB (342V, 20Ah) \\
\hline & I/S: A 2.6, B 2.636 \\
Gear ratio & S/O: A 2.2432 \\
& S/P: R1 0.882, R2 1.97, R3 0.575 \\
\hline Final Gear & 3.5 \\
\hline
\end{tabular}

\subsubsection{Engine / Motor / Battery Modelling}

The efficiency of engine and motor developed at Hyundai Motors are measured in-house. 


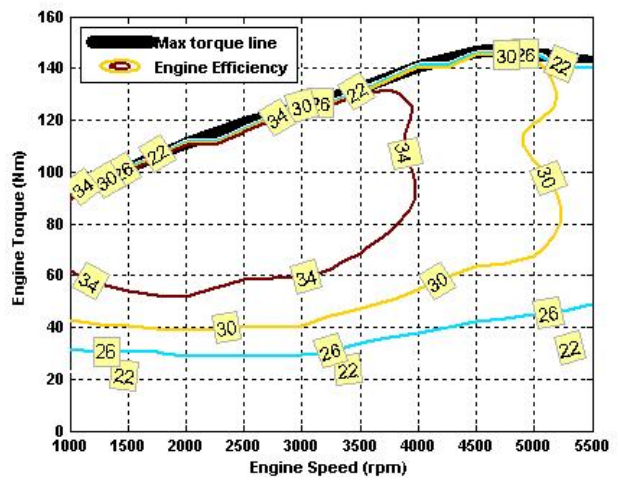

Figure7: Efficiency map of 1.6L gasoline engine

The base efficiency maps of motor and generator efficiency also measured in-house are shown in Figure 7. They are $39 \mathrm{~kW}$ Permanent Magnet Synchronous Machine (PSMS) respectively and they are scaled up or down depending on PHEV system type. The average efficiency of motor and generator of each system is assumed to same because the influence of motor and generator operating point is not considered in this study.
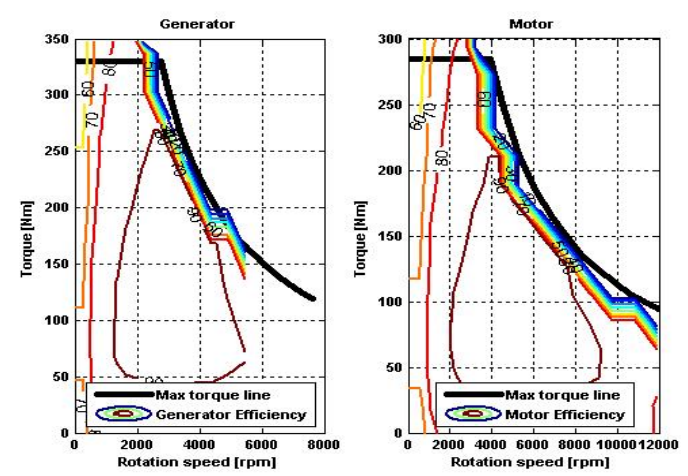

Figure8: Efficiency map of scaled Motor /Generator

Table3: Motor/Generator max power of PHEVs

\begin{tabular}{|c|c|}
\hline System & Generator / Motor power \\
\hline Input Split & $42 \mathrm{~kW} / 60 \mathrm{~kW}$ \\
\hline Series-Output Split & $55 \mathrm{~kW} / 111 \mathrm{~kW}$ \\
\hline Series-Parallel & $100 \mathrm{~kW} / 124 \mathrm{~kW}$ \\
\hline
\end{tabular}

The battery used in this study as the reference is Lithium-ion Polymer Battery (LiPB) that is measured in house and a simple battery state-ofcharge (SOC) model is derived from the equivalent circuit model as follows [15]:

$$
S O C=-\frac{V_{O C}-\sqrt{V_{O C}{ }^{2}-4 P_{\text {Batt }} R_{\text {Batt }}}}{2 R_{\text {Batt }} Q}
$$

Where Voc is open circuit voltage, RBatt is the internal resistance of the battery, $\mathrm{Q}$ is the battery capacity, and PBatt is the net power drawn by the two electric machines.

\subsubsection{Transmission Modelling}

Among the mechanical losses, three primary elements - gearset, clutch and oil pump losses are considered. Input split has normally two planetary gears, one as a power split device and one as a motor reduction gear. Series-output split has one planetary gear and three clutch elements and Series-parallel has three spur gear and one clutch element.

Table4: Mechanical Components of PHEV systems

\begin{tabular}{|c|c|c|c|c|}
\hline \multicolumn{2}{|c|}{$\begin{array}{c}\text { Mechanical } \\
\text { Components }\end{array}$} & Input split & $\begin{array}{c}\text { Series- } \\
\text { Output }\end{array}$ & $\begin{array}{c}\text { Series- } \\
\text { Parallel }\end{array}$ \\
\hline \multirow{2}{*}{ Gear } & PG & 2 & 1 & 0 \\
\cline { 2 - 5 } & SG & 0 & 0 & 3 \\
\hline \multicolumn{2}{|c|}{ Clutch } & 0 & 3 & 1 \\
\hline
\end{tabular}

The spur gear efficiency can be considered as a constant value, 99\%. A planetary gear set efficiency of power split is in the range of $96.7 \%$ to $97.0 \%$ regardless of difference in torque and speed conditions [16, 17].

Clutch loss is caused by oil drag between friction disk and retainer when it is disengaged. While the mathematical modelling of clutch loss is impossible, the experimental model is used.

$$
T_{\text {Loss,Clutch }}=f\left(N_{\text {Friction, }} \Delta \omega\right)
$$

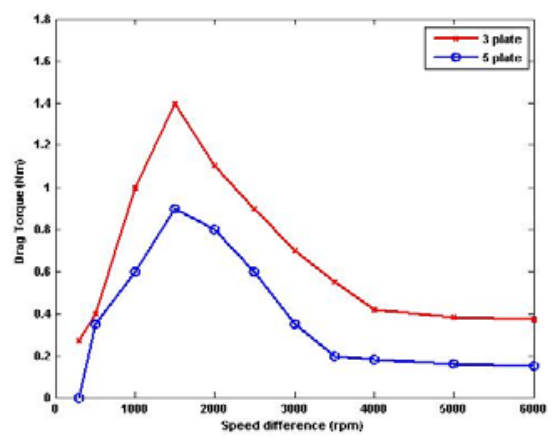

Figure9: Clutch loss model

Each PHEV system require oil pump for lubrication, cooling and clutch operation. Oil pump drag is the function of oil pump speed and oil pump displacement, which is related to the number of operating clutch. The equation of oil pump drag can be expressed as follows.

$T_{\text {Loss,OilPump }}=f\left(\omega_{\text {OilPump }}\right.$, NumberofClutch $)$

Since it has no clutch operation, Input split requires small oil pump capacity and low oil pump drag. On the other hand, since having three clutches and one clutch respectively, Series-Output split and Series-Parallel have large oil pump drag proportionally. The drag of two kinds of oil pump - no clutch operation and 4 clutches operation are measured in-house and one clutch and three clutch operation oil pump are modelled. 


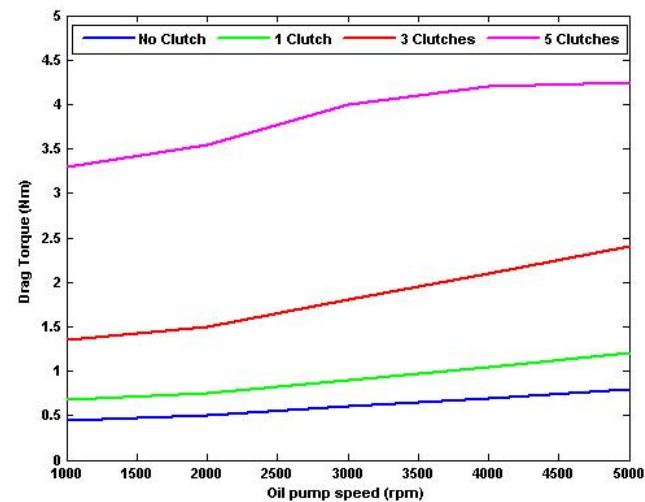

Figure10: Oil pump loss model

\subsection{Dynamic Programming}

HEV / PHEV are the type of vehicle which combines a conventional internal combustion engine and with an electric motor, therefore the energy management of two traction system is a critical problem to reduce fuel consumption. Dynamic programming (DP) technique is an effective tool to find the globally optimal use of multiple energy sources over a predefined drive cycle [18]. The results of DP for PHEV systems can be used as follows:

- Finding maximum fuel economy potential

- Finding a optimal control strategy

- Finding a optimal component size

In this study the maximum system efficiency in a certain drive cycle of each PHEV system are compared by using deterministic dynamic programming. Deterministic dynamic programming can be expressed in discrete forms as follows. The discrete time system modelling and state variable / control signal constrains are shown in Eq. (7) and Eq. (8) respectively.

$x_{k+1}=f\left(x_{k}, u_{k}\right), k=0,1, \cdots, N-1$

$x(t) \in X(t), u(t) \in U(t)$

Where $\boldsymbol{x}_{\boldsymbol{k}}$ is the state variable e.g. SOC and $\boldsymbol{u}_{\boldsymbol{k}}$ is the control signal e.g. gear mode or power distribution for PHEV. $\boldsymbol{X}$ is SOC constraint and $\boldsymbol{U}$ is the component constraint such as engine, motor or battery for PHEV. The examples of the control vector in "Series+ $\boldsymbol{\alpha}$ " PHEVs are as follows.

Table5: DP's Control Vectors of PHEV systems

\begin{tabular}{|c|c|}
\hline PHEV systems & Control Vector \\
\hline Input Split & Engine Speed / Torque \\
\hline Series-Output Split & $\begin{array}{c}\text { Gear mode / Engine Speed / } \\
\text { Engine Torque }\end{array}$ \\
\hline Series-Parallel & $\begin{array}{c}\text { Gear mode / Engine Speed / } \\
\text { Engine Torque }\end{array}$ \\
\hline
\end{tabular}

Let the discretized cost with the initial state $\boldsymbol{x}(0)=\boldsymbol{x}_{0}$ be

$$
\begin{aligned}
J_{\pi}\left(x_{0}\right)= & g_{N}\left(x_{N}\right)+\Phi_{N}\left(x_{N}\right) \ldots \\
& +\sum_{k=0}^{N-1}\left[h_{k}\left(x_{k}, u_{k}\right)+\Phi_{k}\left(x_{k}\right)+\alpha \times \Delta x\right]
\end{aligned}
$$

Where $\boldsymbol{g}$ and $\boldsymbol{h}$ is the cost function, $\Phi$ is the penalty function by constraints and $\boldsymbol{\alpha}$ is the fuelbattery energy conversion coefficient. For CS mode the initial and final SOC is same by state constraint penalty, on the other hand for CD mode SOC consumption is considered in cost function by fuel-battery conversion coefficieny ' $\alpha$ ' as well. The optimal control policy is the policy that minimizes $\boldsymbol{J}_{\boldsymbol{\pi}}$ :

$$
\boldsymbol{J}^{0}\left(\boldsymbol{x}_{0}\right)=\min \boldsymbol{J}_{\boldsymbol{\pi}}\left(\boldsymbol{x}_{0}\right)
$$

Based on the principle of optimality [19], dynamic programming is the algorithm which evaluates the optimal cost-to-go function $J_{\pi}\left(x^{i}\right)$ at every node in the discretized state-time space by proceeding backward in time:

- End cost calculation step

$$
J_{N}\left(x^{i}\right)=g_{N}\left(x^{i}\right)+\Phi_{N}\left(x^{i}\right)
$$

- Intermediate calculation step for $\mathrm{k}=\mathrm{N}-1$ to 0

$$
\begin{aligned}
J_{k}\left(x^{i}\right)= & \min _{u_{k} \in U_{k}} \mid h_{k}\left(x^{i}, u_{k}\right)+\Phi_{N}\left(x^{i}\right) \ldots \\
& \ldots+\alpha \times \Delta x_{k}+J_{k+1}\left(F_{k}\left(x^{i}, u_{k}\right)\right) \mid
\end{aligned}
$$

The optimal control is given by the argument that minimizes the right-hand side of Eq. (12) for each $\boldsymbol{x}^{i}$ at time index $\boldsymbol{k}$ of the discretized state-time space [20]. With the optimal control from backward simulation, the optimal fuel economy can be found by forward simulation.

\section{System efficiency of PHEV systems in steady state}

\subsection{Definition of PHEV system efficiency}

PHEV system efficiency can be divided by Engine system efficiency and Transmission (TM) system efficiency. Engine system efficiency consists of Engine component efficiency and Parallel-path efficiency, and Transmission system efficiency consists of Transmission Mechanical efficiency and Transmission Electric efficiency. The definition and explanation of efficiency is summarized below:

- TM Mechanical Efficiency is the ratio of TM mechanical loss power to engine power. It is occurred when power is transmitting through 
gearset, clutch is disengage or oil pump is spinning

- TM Electric Efficiency, or Series-path efficiency, is the ratio of electric machine loss power to engine power. It is occurred when one electric machine is generating and the other one is motoring. TM Electric efficiency of Input split and Output split is depending on speed ratio, and that of Series and Parallel is the multiplication of efficiency of Electric machine and 100\% respectively.

- Parallel-path Efficiency is the ratio of highvoltage battery loss power to engine power. It is occurred when part of engine power goes through high-voltage battery such as driving charging condition. Mostly, the purpose of driving charging is for SOC balancing or engine operating control.

In this study, Engine system efficiency is defined by the multiplication of Engine component efficiency and Parallel-path efficiency. Because mostly, parallel-path is a result of engine optimal control to improve engine component efficiency. Thus engine component efficiency and parallelpath efficiency have a trade-off relationship.

TM system efficiency is defined by the multiplication of TM mechanical efficiency and TM electric efficiency. When it comes to PHEV, the loss of Electric machine can be considered that of TM, in that electric machines can be considered parts of TM. The energy path flow of PHEV is shown in Figure 11.



Figure11: Energy Flow schematic of PHEV system

The equation of TM electric efficiency and Parallel-path efficiency are summarized below:

$\eta_{T M_{-} \text {Elec }}=1-(1-\alpha) \cdot \boldsymbol{\beta} \cdot\left(1-\eta_{1} \cdot \eta_{2}\right)$

$\eta_{\text {Par-path }}=1-(1-\alpha) \cdot(1-\beta) \cdot\left(1-\eta_{1} \cdot \eta_{2} \cdot \eta_{H V}\right)$

$\alpha=\frac{A}{A+B+C}, \beta=\frac{B}{B+C}$
Where A is engine direct power, B is series-path power, $\mathrm{C}$ is parallel-path power, and $\eta$ is efficiency. 1, 2, and HV are subscript for MG1, MG2 and High-voltage battery respectively.

\subsection{Theoretical Transmission Electric efficiency of PHEV}

The theoretical TM electric efficiency (Series-path efficiency) of Series, Parallel, Input split, and Output split can be compared by system equation. Assuming no battery power and the efficiency of electric machines is constant, 90\%, TM electric efficiency and split power ratio can be calculated from system equation.

For Input split, system equation, TM electric efficiency and split power ratio are summarized below.

$$
\begin{aligned}
& T_{i}=-(A+1) T_{1} \\
& T_{o=} \frac{A}{A+1} T_{i}+T_{2} \\
& \omega_{i=} \frac{1}{A+1} \omega_{1}+\frac{A}{A+1} \omega_{o} \\
& \frac{\omega_{1} T_{1}}{\eta_{1}}+\frac{\omega_{2} T_{2}}{\eta_{2}}=0 \\
& \eta_{T M-E l e c}=\frac{1}{\rho}\left\{\frac{\eta_{2}}{\eta_{1}}\left(\boldsymbol{\rho}-\rho_{\boldsymbol{e} 1}\right)+\rho_{\boldsymbol{e} 1}\right\} \\
& \Phi_{1}=\left(\frac{\boldsymbol{\rho}_{\boldsymbol{e} 1}}{\boldsymbol{\rho}}-1\right) \\
& \rho_{e 1}=\frac{1}{A+1} ; \text { Mechanical point for Input split }
\end{aligned}
$$

Where $\mathrm{A}$ is the ratio of gear teeth between the sun and the ring of a planetary gear, $T$ and $\omega$ are torque and speed. $i, o, 1$ and 2 are subscript for engine, output, MG1 and MG2 respectively. $\eta, \rho$, $\Phi$ are efficiency, speed ratio and split power ratio respectively.

For Output split, system equation, TM electric efficiency and split power ratio are summarized below.

$$
\begin{aligned}
& T_{i}=-T_{1}+A \times T_{2} \\
& T_{o=}(A+1) T_{2} \\
& \omega_{2=}-A \times \omega_{i}+(A+1) \omega_{o} \\
& \frac{\omega_{1} T_{1}}{\eta_{1}}+\frac{\omega_{2} T_{2}}{\eta_{2}}=0 \\
& \eta_{\text {TM-elec }}=\frac{1}{\boldsymbol{\rho}} \frac{1}{\left\{\frac{\boldsymbol{\eta}_{2}}{\boldsymbol{\eta}_{1}}\left(\frac{1}{\boldsymbol{\rho}}-\frac{1}{\boldsymbol{\rho}_{\boldsymbol{e} 2}}\right)+\frac{1}{\boldsymbol{\rho}_{\boldsymbol{e} 2}}\right\}}
\end{aligned}
$$


$\Phi_{2}=\frac{1}{\left(\frac{\eta_{2}}{\eta_{1}} \times \frac{\rho}{\rho-\rho_{\boldsymbol{e} 2}}-1\right)}$

$\rho_{e 2=} \frac{A+1}{A}$; Mechanical point for Output split

TM Electric efficiency of Input split and Output split are varied depending on speed ratio. The more power ratio of the generator over engine, the less TM electric efficiency is. And at the mechanical point, TM electric efficiency is $100 \%$ in that there is no power split. Normally, engine power is divided with output and generator, however on a certain speed range, motor is generating while generator is motoring, which is called recirculation. In a recirculation condition, engine power circulates through electric machines and this leads to lower TM electric efficiency.

TM Electric efficiency of Series and Parallel are the product of two motors efficiency, $81 \%$ and $100 \%$ respectively, and split power ratio of them are $100 \%$ and $0 \%$ respectively in the condition of no battery power.

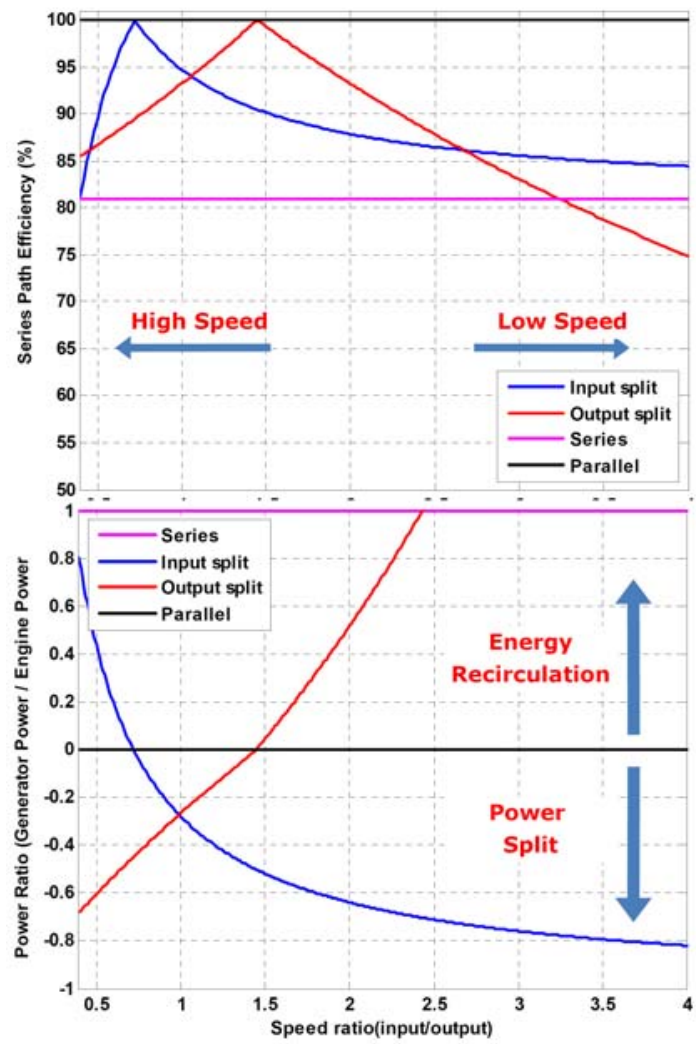

Figure12: TM electric efficiency and Power ratio

The meaningful notifications of analysis of the theoretical TM electric efficiency are summarized below:
- Input split has a mechanical point at a low speed ratio (0.772) and Output split has it at a middle speed ratio (1.45).

- Input split has energy recirculation at low speed ratio range (high speed) and Output split has it at high speed ratio range (low speed).

- Since, in series mode, all engine power transmits through electric machine, TM electric efficiency is the multiplication of two electric machines' efficiencies. And, in parallel mode, TM electric efficiency is $100 \%$ because engine power directly flows to the wheel in the condition of no charging battery.

The theoretical TM electric efficiency of each Plug-in system - Input split, Series-output split and Series-parallel - is compared and speed ratio distribution of each vehicle from UDDS City cycle test is shown in Figure 13. The notifications from this analysis are summarized below.

- At low speed range (city driving), Input split has an advantage over Series-Output split and Series-Parallel system, however mostly engine in that speed turns off. Thus the merit of Input split is effective when engine turns on frequently such as low SOC condition or traffic congestion.

- TM electric efficiency of it is lower than $80 \%$ at low speed range, Series-output split covers with EV partly. In addition it is expected to have better efficiency in suburban driving, because its mechanical point is at middle speed range.

- Even though it has poor TM electric efficiency in series mode its EV ability covers most broadly, so in the condition of mild city driving Series-parallel can have better efficiency than other systems. On the other hand, its efficiency drops dramatically when series is selected such as low SOC or uphill condition. Plus because of Parallel mode, it also has an advantage over other system in mild highway driving. 

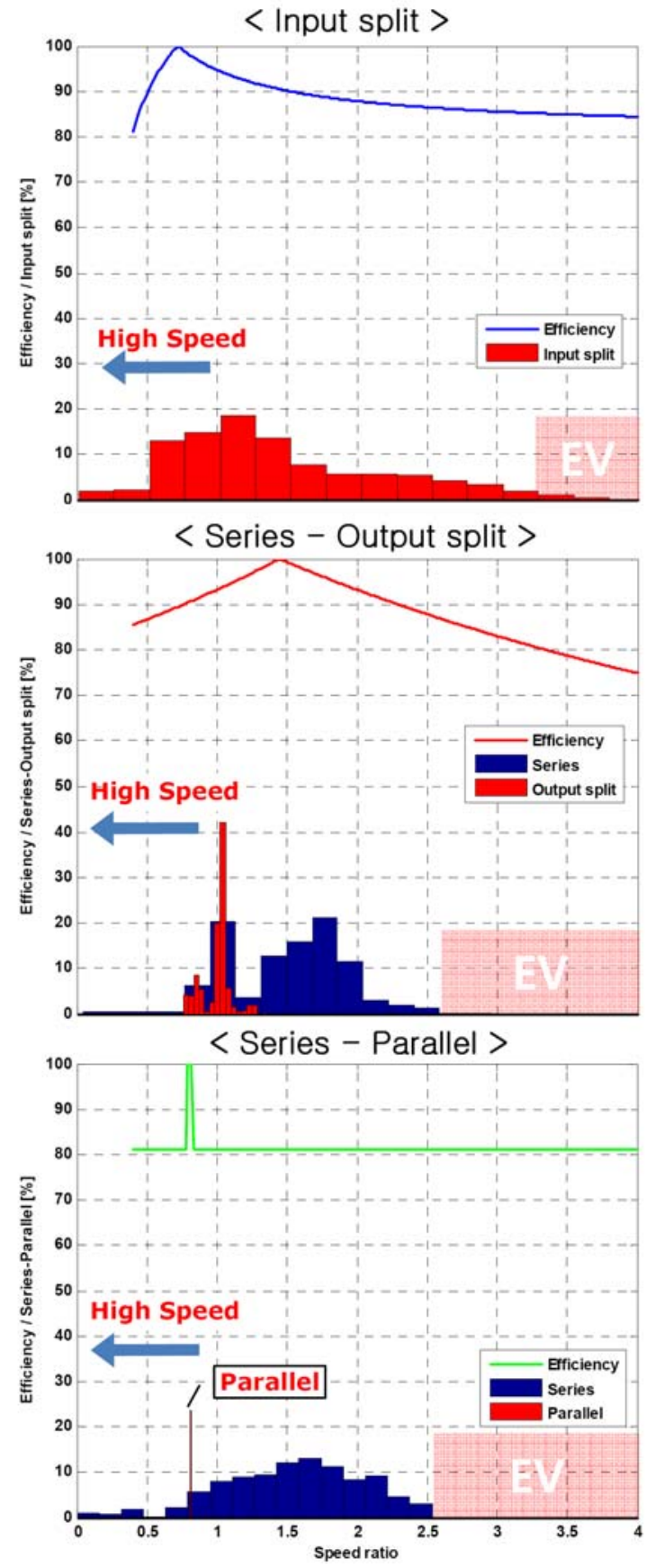

Figure13: TM electric efficiency and Speed ratio distribution of Plug-in vehicles in city cycle

\subsection{Theoretical Transmission Mechanical efficiency of PHEV}

The planetary gearset loss of Input split and Output split is related to the ratio of split power and the length of power transfer path. Since the power ratio between the engine and motor/generator is the function of the speed ratio, the transmission mechanical efficiency is the function of the speed ratio as well.

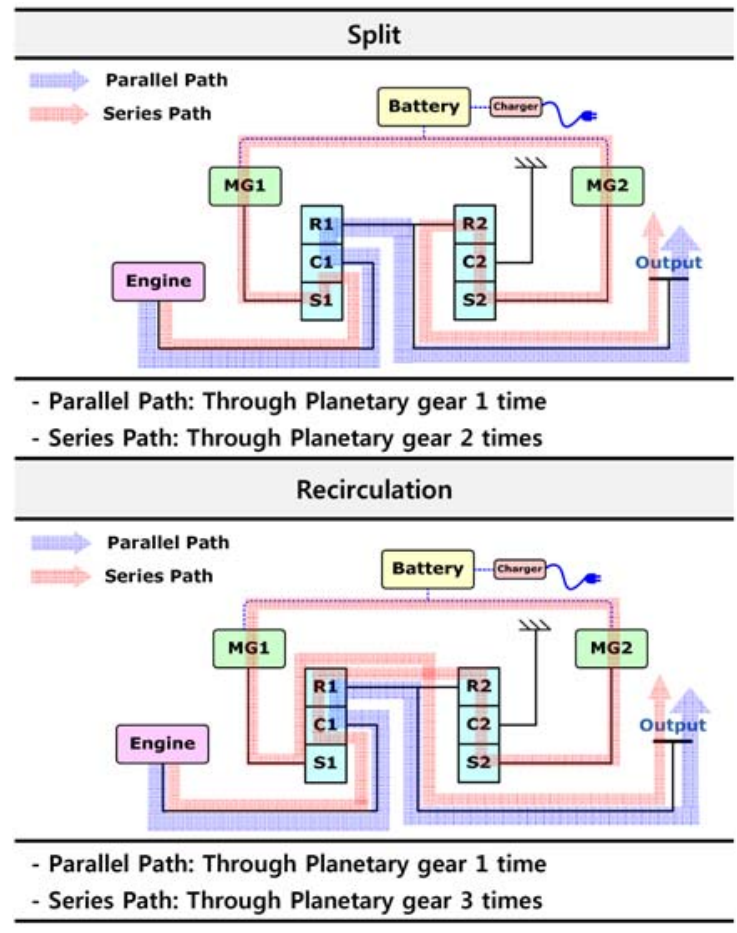

Figure14: Split and Recirculation path of Input split

As shown in Figure 14 when it comes to Input split system, Parallel path has planetary gear loss one time and Series path does it two times in Power split mode, while Series path does three times in Power recirculation mode. Therefore the planetary gearset loss in power recirculation mode increases dramatically. On the assumption that no battery power, no motor/generator loss and same planetary gearset efficiency without reference to power path (e.g. carrier-to-sun or carrier-to-ring), the equation of the theoretical TM mechanical efficiencies of Input split system in split and recirculation mode are shown as below.

$\eta_{T_{-} m_{-} \text {split }}=\frac{\left(\boldsymbol{P}_{\boldsymbol{i}}+\boldsymbol{P}_{1}\right) \cdot \boldsymbol{\eta}_{\mathbf{P G 1}}-\boldsymbol{P}_{1} \cdot \boldsymbol{\eta}_{\mathbf{P G 1}} \cdot \boldsymbol{\eta}_{\mathbf{P G 2}}}{\boldsymbol{P}_{\boldsymbol{i}}}$

$=\left(1+\Phi_{1}\right) \cdot \eta_{P G 1}-\Phi_{1} \cdot \eta_{P G 1} \cdot \eta_{P G 2}$

$\eta_{T M_{-} m_{-} \text {Recir }}=\frac{\left(\boldsymbol{P}_{\boldsymbol{i}}-\boldsymbol{P}_{1}\right) \cdot \boldsymbol{\eta}_{P G 1}+\boldsymbol{P}_{1} \cdot \boldsymbol{\eta}_{P G 1}{ }^{2} \cdot \boldsymbol{\eta}_{P G 2}}{\boldsymbol{P}_{\boldsymbol{i}}}$

$=\left(1-\Phi_{1}\right) \cdot \eta_{P G 1}+\Phi_{1} \cdot \eta_{P G 1}^{2} \cdot \eta_{P G 2}$

$\eta, \mathrm{P}, \Phi$ are efficiency, speed ratio and Split power ratio respectively. And $i, 1$ and PG are subscript for engine, output, MG1 and Planetary gear respectively. 



- Parallel Path: Through Planetary gear 1 time - Series Path: Through Planetary gear 2 times

Figure15: Split and Recirculation path of Output split

As shown in Figure 15 when it comes to Output split system, Parallel path has planetary gear loss one time and Series path has it two times in Power split mode, while Series path has it two times in Power recirculation mode. The equation of the theoretical TM mechanical efficiencies of Output split system in power split and power recirculation mode is shown as below.

$$
\begin{aligned}
& \eta_{T M_{-} \boldsymbol{m}_{-} s p l i t}=\frac{\left(\boldsymbol{P}_{\boldsymbol{i}}+\boldsymbol{P}_{1}\right) \cdot \eta_{P G}-P_{1} \cdot \eta_{P G}}{\boldsymbol{P}_{\boldsymbol{i}}} \\
& =\left(1+\Phi_{1}\right) \cdot \eta_{P G}-\Phi_{1} \cdot \eta_{P G}=\eta_{P G} \\
& \eta_{\boldsymbol{T} M_{-} \boldsymbol{m}_{-} \text {Recir }}=\frac{\left(\boldsymbol{P}_{\boldsymbol{i}}-\boldsymbol{P}_{1}\right) \cdot \eta_{P G}+\boldsymbol{P}_{1} \cdot \eta_{P G}{ }^{2}}{\boldsymbol{P}_{\boldsymbol{i}}} \\
& =\left(1-\Phi_{1}\right) \cdot \eta_{P G}+\Phi_{1} \cdot \eta_{P G}{ }^{2}
\end{aligned}
$$

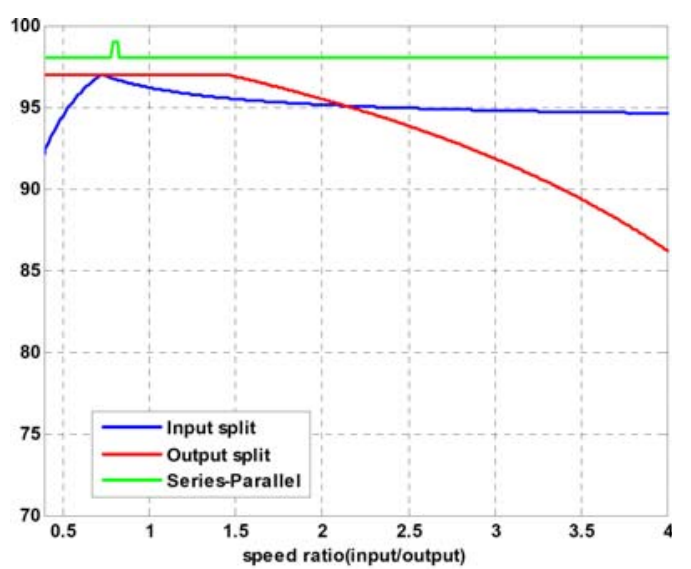

Figure16: The theoretical TM Mechanical efficiency of PHEV systems
With respect to gear loss, series mode has advantage over input split and output split mode owing to high efficiency of spur gear. Among split system, input split has high efficiency in high speed ratio (low speed) while so does output split in low speed ratio (high speed).

\subsection{Theoretical Transmission System efficiency of PHEV}

The energy path and the mechanical loss of Charging depleting (CD) mode and Charging sustaining (CS) mode are different. Therefore the strength and weakness of PHEV systems are depending on CD/CS mode and driving condition.

\subsubsection{Charging Depleting (CD) mode}

In electric driving condition, Series-parallel has short power transfer path and high mechanical efficiency thanks to spur gear. When it comes to Input split, generator is connected to a planetary gear while electric driving, which leads to additional generator inertia loss. Hence Seriesparallel has the highest system efficiency, Seriesoutput is just behind it and Input-split follows in electric driving condition.



Figure17: Power transfer path of EV of PHEV systems

Table6: Mechanical Component of PHEV systems

\begin{tabular}{|l|c|c|c|c|}
\hline \multicolumn{2}{|c|}{ Efficiency } & I/S & S/O & S/P \\
\hline \multirow{2}{*}{ Mech. } & Gear & $97 \%$ & $97 \%$ & $99 \%$ \\
\cline { 2 - 5 } & MG drag & $0 \sim 1 \%$ & 0 & 0 \\
\hline \multicolumn{2}{|c|}{ Electrical loss } & \multicolumn{3}{|c|}{$90 \%$ (Assumption) } \\
\hline \multicolumn{2}{|c|}{ System } & $\mathbf{8 6 ~ 8 7 ~ \% ~}$ & $\mathbf{8 7} \%$ & $\mathbf{8 9 \%}$ \\
\hline
\end{tabular}

Basically, the purpose of series mode of S/O and $\mathrm{S} / \mathrm{P}$ is expanding EV range. Plus thanks to Series mode, vehicle can be operated when battery power is limited e.g. cold weather.

\subsubsection{Charging Sustaining (CS) mode}

The theoretical system transmission of each system in HEV mode is the multiplication of TM Electric efficiency and TM mechanical efficiency. Even though Input split has the highest efficiency in low/high speed range and Output split has strength in middle speed range as shown in Figure 18, the comparison of combined PHEV systems is complex because of parallel and EV mode. 
While it has low efficiency in series mode, $\mathrm{S} / \mathrm{P}$ is advantageous in parallel an EV mode. Moreover high efficiency in EV mode is equal to high efficiency in regeneration mode, which leads to extend electric driving area. Hence in a mild driving condition when EV and Parallel are frequent, Series-parallel reaps benefit over other systems. On the other hand, in a congestion condition - high acceleration or hill climbing condition - it operates on series mode, thus it has poor system efficiency.

In addition in high load condition, series mode of $\mathrm{S} / \mathrm{P}$ can have better system efficiency that parallel mode. Because parallel-path efficiency decrease due to increase the amount of battery charging power in order to operate engine on optimal operating line (OOL). Series can be advantageous over parallel, since series mode just transmits through electric machines while parallel mode does through electric machines and battery.



Figure18: The theoretical TM System efficiency of PHEV systems

Series-output split has better efficiency over Input split in low speed range but in high speed range vice versa. Furthermore, Series-output split has low efficiency in a congestion and high load condition because of series mode. In addition the tractive effort of the powerflow of output split and parallel is limited, therefore Series driving is needed in that case [14].

The other transmission component loss such as clutch and oil pump can affect to system efficiency as well like the motor/generator efficiency. These aspects will be discussed in the next section.

\section{System efficiency of PHEV systems in known trajectories}

\subsection{Validation}

Even though the specifications of vehicle and simulation are different the dynamic programming results can be validated by comparing the characteristic of driving mode. Prius PHEV, Volt and Accord PHEV UDDS City test results are used for comparison.


Figure19: Comparison Input split DP result and Prius PHEV test results in city driving

The driving mode distributions of test and DP of I/S are similar except for high speed. According to the city test result, the maximum EV speed of Prius PHEV is $60 \mathrm{~km} / \mathrm{h}$. However it is reported that maximum EV speed is $100 \mathrm{~km} / \mathrm{h}$, so the DP follows it [21].

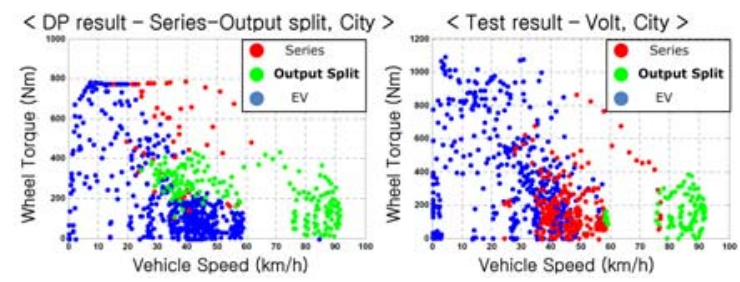

Figure20: Comparison Series-output split DP result and Volt test results in city driving

When it comes to Series-output split, Series mode is selected at middle speed and high torque and Output split be at high speed and low torque. Compared to Volt test result, DP result has more Output split mode. This can be because of the difference between the optimal solution and the rule-based control of production vehicle, and SOC balance in real driving condition.

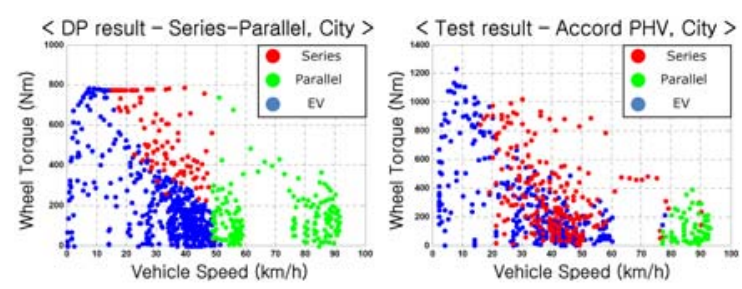

Figure21: Comparison Series-Parallel DP result and Accord PHEV test results in city driving 
When it comes to Series-parallel, the boundary between Series and Parallel is determined by engine minimum speed, 1300rpm in this case. In the test result, it is $77 \mathrm{~km} / \mathrm{h}$ and that means the minimum engine speed of parallel mode is different and the SOC balance also affects to mode selection. In this study, for the comparison of PHEV system the minimum engine speed is setting to 1300rpm altogether.

\subsection{Result}

\subsubsection{Charging Depleting (CD) mode}

When it comes to CD mode, the transmission mechanical efficiency is critical for PHEV if the motor efficiency is assumed to same. The simulation results of $\mathrm{CD}$ mode are shown in Table 7.

Table7: TM mechanical efficiency in CD mode

\begin{tabular}{|c|c|c|c|}
\hline Efficiency (\%) & I/S & S/O & S/P \\
\hline City & 96.0 & 96.1 & 96.9 \\
\hline HWY & 96.0 & 97.0 & 97.5 \\
\hline
\end{tabular}

Thanks to the shorter mechanical path, Seriesparallel has better TM mechanical efficiency and Input split is disadvantageous with respect to the generator inertia loss and planetary gearset loss.

\subsubsection{Charging Sustaining (CS) mode}

The DP of CS mode is performed on City, Hwy and US06 certification cycle. The dynamic programming results of CS mode are shown in Table 8. The engine system efficiency is the multiplication of Engine component efficiency and Parallel-path efficiency and the Transmission system efficiency is the multiplication of TM mechanical efficiency and TM electric efficiency as defined in section 4.1.

Table8: Powertrain system efficiency in CS mode

\begin{tabular}{|c|c|c|c|}
\hline Efficiency (\%) & I/S & S/O & S/P \\
\hline $\begin{array}{c}\text { City, System Eff. } \\
\text { Eng. system }\end{array}$ & 31.8 & 31.0 & 30.9 \\
\hline TM system & 89.2 & 34.5 & 34.6 \\
\hline HWY, System Eff. & 33.2 & 90.0 & 89.3 \\
\hline Eng. system & 35.2 & 35.4 & 33.4 \\
\hline TM system & 94.4 & 85.0 & 34.9 \\
\hline $\begin{array}{c}\text { US06, System Eff. } \\
\text { Eng. system }\end{array}$ & 34.1 & 32.2 & 33.9 \\
\hline TM system & 92.9 & 36.4 & 35.1 \\
\hline
\end{tabular}
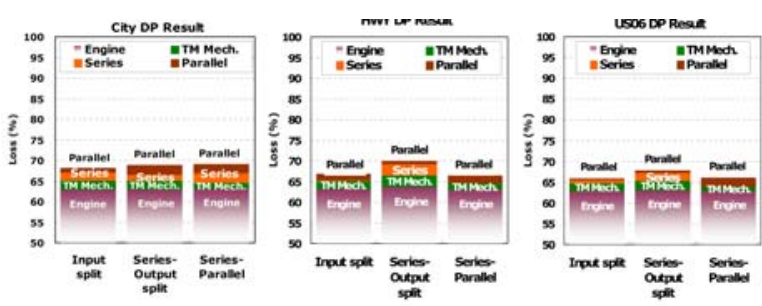

Figure22: DP results of Input split, Series-output split and Series-parallel in City, HWY and US06 cycle

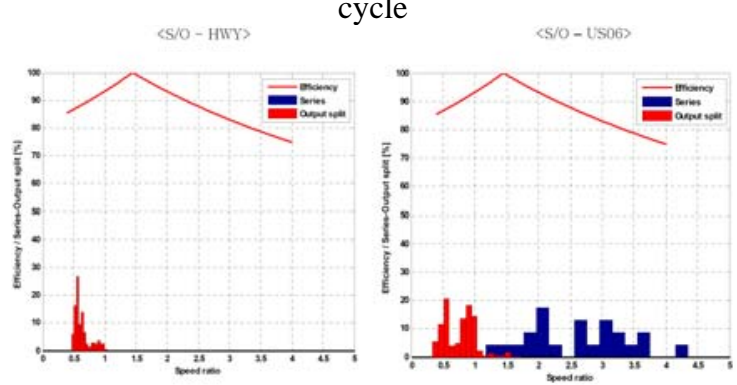

Figure23: Theoretical Transmission efficiency and speed ratio distribution of Series-output split in HWY and US06 cycle

The conclusions of DP results are shown as follows:

- TM Electric efficiency of Series-output split in HWY and US06 cycles is low since it has more power split ratio than Input split and Series-parallel owing to gear ratio.

- In city cycle, Input split has higher powertrain efficiency because of high parallel-path efficiency. On the other hand, parallel have low parallel efficiency because of its parallel mode. In parallel of $\mathrm{S} / \mathrm{P}$, it increase the engine torque to optimal point (Load levelling) and charge the battery.

- In highway and US06 cycle the efficiency of Input split and Series-parallel is similar. Even though $\mathrm{S} / \mathrm{P}$ has low parallel efficiency it is advantageous with respect to series and mechanical efficiency.

\section{Conclusion and Future work}

\subsection{Conclusion}

In this study, the system efficiency of PHEV systems - Input split, Series-output split and Series-parallel - is compared in a steady state condition and a variety of driving condition.

At first the transmission electric and mechanical efficiency of each system in a steady state is expressed in terms of speed ratio. In Charging Depleting (CD) mode, the system efficiency is determined by transmission mechanical efficiency 
on the assumption that motor efficiency and the amount of regeneration of each system are same. Therefore Series-parallel is advantageous over Series-output split and Input-split is last since spur gear of S/P has higher efficiency over I/P and $\mathrm{S} / \mathrm{O}$ is disconnected generator by clutch unlike I/P.

In Charging Sustaining (CS) mode, I/S has the mechanical point at low speed ratio (high speed) and $\mathrm{S} / \mathrm{O}$ has it at under drive (UD) condition. And $\mathrm{S} / \mathrm{P}$ has the highest efficiency in over drive (OD) condition. Hence S/P reaps benefit in high speed and steady condition such as freeway and I/S is better in low speed or high load condition such as congestion, hill or aggressive driving. $\mathrm{S} / \mathrm{O}$ has a merit in UD driving condition like suburban.

On top of that, in order to compare the system efficiency in a certain driving condition, the transmission mechanical models are applied to the dynamic programming (DP). It is verified that the mechanical losses in the function of speed ratio or input speed can change the results of optimal control. In $\mathrm{CD}$ mode, $\mathrm{S} / \mathrm{P}$ is advantageous $1 \sim 1.5 \%$ over $\mathrm{I} / \mathrm{S}$ in terms of transmission mechanical efficiency and $\mathrm{S} / \mathrm{O}$ be $0 \sim 1 \%$ over I/S. In CS mode, I/S has 2 3\% better system efficiency over S/O and S/P for City cycle. For highway and US06 cycle I/S and S/P have similar efficiency and $\mathrm{S} / \mathrm{P}$ is last.

To sum up, for PHEVs which put more weight on electric driving, Series-parallel is better than Series-Output split and Input split follows. For PHEVs which put more weigh on hybrid driving, Input split and Series-parallel have a merit over Series-output split.

\subsection{Future work}

- Each system may have optimal gear ratio and engine/motor/generator power and the specification of each system can affect the system efficiency. Next research would find the optimal gear ratio and components power and whether it affects the system efficiency.

- In this study, the average efficiency of motor/generator is assumed same. However, the more power the motor has, the better efficiency it has. Hence PHEV systems which require bigger motor or generator capacity - Series-output split and Seriesparallel may have better electric machines component efficiency.

\section{Acknowledgments}

The author would like to acknowledge Heera Lee, Jane Kim and Jinsook Mook for their support and comment for this study.

\section{References}

[1] Lawrence Ulrich, Carbon Car, IEEE Spectrum, 2013, 30-31

[2] K. C. Bayindir et. Al., SUBAT, A comprehensive overview of hybrid electric vehicle: Powertrain configurations, powertrain control techniques and electronic control units, Energy Conversion and Management 52 (2011), 1305-1313

[3] Xin Li et. Al., Comparative Investigation of Series and Parallel Hybrid Electric Vehicle (HEV) Efficiencies Based on Comprehensive Parametric Analysis, Vehicle Power and Propulsion Conference (VPPC), IEEE, 2008

[4] Yimin Gao et. Al., Design and Control Methodology of Plug-in Hybrid Electric Vehicles, IEEE Transactions on Industrial Electronics, vol. 57, No. 2, 2010

[5] Yanhe Li et. Al., Advanced Design Approach of Power Split Device of Plug-in Hybrid Electric Vehicles Using Dynamic Programming, Vehicle Power and Propulsion Conference (VPPC), IEEE, 2011

[6] Dominik Karabowski et. Al., Plug-in Vehicle Control Strategy: From Global Optimization to Real-time application, EVS22, 2006

[7] O. Torres et. Al., Comparison of different Hybrid Electric Vehicles Concepts in terms of Consumption and Efficiency, EVS26, 2012

[8] Brendan Conlon., Comparative Analysis of Single and Combined Hybrid Electrically Variable Transmission Operating Modes, SAE technical paper 2005-01-1162, 2005

[9] A. Da Costa et. Al., Fuel Consumption Potential of Different Plug-in Hybrid Vehicle Architectures in the European and American Contexts, EVS26, 2012

[10] Chao Ma et. Al., Comparative Study on Power Characteristics and Control Strategies for Plug-in HEV, Vehicle Power and Propulsion Conference(VPPC), IEEE, 2011

[11] Antonio Sciarretta et. Al., Control of Hybrid Electric Vehicles, IEEE Control Systems MAgazine, IEEE, 2007

[12] Bram de Jager et. Al., Optimal Control of Hybrid Vehicles, ISBN 978-1-4471-5075-6, Springer, 2011 
[13] E. D. Tate et. Al., The Electrification of the Automobile: From Conventional Hybrid, to Plug-in Hybrids, to Extended-Range Electric Vehicles, SAE technical paper 2008-01-0458, 2008

[14] Michael A. Miller et. Al., The GM "Voltec" 4ET50 Multi-Mode Electric Transaxle, SAE technical paper 2011-01-0887, 2008

[15] X. Zhang, C. Li, D. Kim, H. Peng, Prius ${ }^{+}$ and Volt: Configuration Analysis of PowerSplit Hybrid Vehicles with a Single Planetary Gear, IEEE Transactions on Vehicular Technology, Vol. 61, No. 61, pp.3544-3552, 2012

[16] T.T. Petry-Johnson et. Al., An Experimental Investigation of Spur Gear Efficiency, Journal of Mechanical Design 130, 2008

[17] H. J. Kim et. Al., Planetary Gearset Lubrication Requirement Estimation Based on Heat Generation, AGMA Technical paper, 2008

[18] Rui Wang, Srdjan M. Lukie, Dynamic Programming Technique in Hybrid Electric Vehicle Optimization, Electric Vehicle Conference (IEVC), 2012

[19] Bellmad Richard, Dynamic Programming, Princeton University Press, Princeton, 1957

[20] O. Sundström et. Al., On Implementation of Dynamic Programming for Optimal Control Problems with Final State Constrains, Oil \& Gas Science and Technology - Rev. IFP, Vol. 65, No. 1, pp. 91-102, 2010

[21] Shizuo Abe, Development of Toyota Plug-in Hybrid Vehicle, Journal of Asian Electric Vehicles, Volume 8, Number 2, 2010

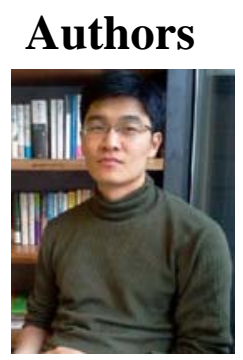

Insup Bill Kim is a research engineer of Advanced Eco-vehicle Development Team at Hyundai-Kia Motor Company. He is developing advanced hybrid system and optimizing system using dynamic programming. He received bachelor's degree in School of Mechanical Engineering from Seoul National University, South Korea, in 2006. He is enrolling for Green-tech MBA at Hanyang university, Korea.



Hyunsup Kim was granted the Ph.D. degree in control engineering from Hanyang University, Seoul, Korea. He is a senior research engineer of Advanced Eco-vehicle Development Team at Hyundai-Kia Motor Company. He is conducting advanced hybrid system development and system optimization using dynamic programming. 